\title{
A Pilot Study of Skin Cancer Risk Reduction Behaviors, Cancer Communication, and Skin Cancer Beliefs in Hispanics
}

\author{
Salina M. Torres ${ }^{1}$, Marcel Ramos ${ }^{2}$, Robert Leverence ${ }^{1}$,Deborah Bowen ${ }^{3}$, Marianne Berwick ${ }^{1}$, \\ and Jennifer Hay ${ }^{2,4}$ \\ ${ }^{1}$ Division of Epidemiology and Biostatistics, University of New Mexico \\ ${ }^{2}$ Department of Psychiatry \& Behavioral Sciences, Memorial Sloan Kettering Cancer Center \\ ${ }^{3}$ Department of Community Health Sciences, Boston University School of Public Health \\ ${ }^{4}$ Memorial Sloan-Kettering Cancer Center, Department of Psychiatry and Behavioral Sciences
}

\begin{abstract}
Purpose: Given rising rates of deadly melanoma skin cancer in Hispanics, the study objective was to examine skin cancer-related risk reduction behaviors and beliefs to dictate content for culturally targeted skin cancer prevention strategies for Hispanics. Methods/Data Source: An anonymous survey was administered to waiting room volunteers in a primary care facility in Albuquerque, New Mexico to assess skin cancer risk reduction behaviors, screening, cancer information seeking and communication, as well as skin cancer beliefs in Hispanics $(n=48)$ and Non-Hispanic Whites $(n=36)$. Results: We found lower levels of sun protection clothing use among Hispanics compared to Non-Hispanic Whites, but comparable use of sunscreen and shade-seeking among these groups. Hispanic ethnicity was the most important predictor of skin cancer misconceptions, with skin cancer information overload and misconceptions reported more often in Hispanics. Conclusions: This study demonstrates the need for culturally relevant information for ethnic minority populations such as Hispanics who have shown an increased risk of presenting with later stage, more aggressive melanoma skin cancer.

(C) 2014 Californian Journal of Health Promotion. All rights reserved.

Keywords: Hispanics, oncology, skin cancer beliefs, risk reduction
\end{abstract}

\section{Introduction}

Melanoma, the most deadly form of skin cancer (SC), is the sixth most common cancer in the United States and is the fourth most common cancer in New Mexico (New Mexico Tumor Registry [NMTR], 2012). Well-known risk factors for SC such as melanoma include light skin and hair phenotype (American Cancer Society [ACS], 2013), yet melanoma rates are increasing in United States Hispanics (Jaimes et al., 2013). Hispanics are more likely than NonHispanic Whites (NHW) to present with thicker tumors (>1mm; $34.5 \%$ vs. 24.9\%), more regional involvement $(12.4 \%$ vs. $8.3 \%)$, and distant disease (6.6\% vs. 3.6\%) all of which contribute to poorer prognosis (Jaimes et al., 2013).
Most melanomas are caused by ultraviolet radiation, so SC risk reduction recommendations include daily sun protection such as sun avoidance, the use of hats and clothing to block UV exposure and the use of sunscreen (ACS, 2013). The American College of Preventive Medicine and the American Cancer Society recommend annual screening of patients who have a family history of melanoma, are $\geq 50$ years old, have fair skin, or have atypical nevi and/or present with $>50$ nevi along with monthly skin self-examinations (ACS, 2013). Several studies have revealed that Hispanics may be largely unaware of their potential SC risks (Coups et al., 2013), which might limit their use of sun protection. Further, rates of physician screening and skin self-examination (SSE) for SC are lower in Hispanics versus NHW (Coups et al., 2013). Addressing the increased prevalence, morbidity, and mortality of 
Torres S.M., Ramos M., Leverence R., Bowen D., Berwick M., Hay J. / Californian Journal of Health Promotion 2014, Volume 12, Issue 1, 95-100.

melanoma in Hispanics will require strategies to increase risk awareness and risk reduction.

This study examined SC risk reduction, screening behaviors, information seeking, and beliefs among Hispanic as compared to NHW individuals drawn from a primarily Englishspeaking primary care population in Albuquerque, New Mexico. New Mexico ranks first in the nation in the proportion of Hispanic residents (42.1\%); the Albuquerque population is 39.9\% Hispanic (U.S. Census Bureau, 2013). Albuquerque receives high levels of year-round sun exposure and is at higher altitude than many other areas of the United States, making it an important location for the development of SC education for Hispanics. Our findings will identify knowledge gaps in our clinic population to plan for the development of such materials in Albuquerque.

\section{Methods}

\section{Design and Sample}

We administered an anonymous survey to adult patient volunteers $(\mathrm{N}=104)$ within the General Internal Medicine clinic of the University of New Mexico (UNM). Inclusion/exclusion criteria were age $\geq 18$ years and willingness to complete a self-administered questionnaire. This pilot study was approved by the Institutional Review Board of UNM.

\section{Measures}

The measures chosen have been widely utilized and previously published. We assessed selfreported racial/ethnic group, age, gender, marital status, educational attainment, income, United States nativity, and presence of a personal and/or family history of SC.

SC risk reduction behaviors: use of sunscreen, long sleeve shirts, hats, shade-seeking, and sunglasses using assessments similar to the CDC Behavioral Risk Factor Surveillance System (BRFSS) questions on individual 5-point scales (Glanz, et al., 2008).

Prior SC screening by a health professional: a face-valid question concerning prior screening (no/yes).
Perceived SC risk: an established assessment on a 4-point scale with verbal anchors was used (Weinstein, 1980).

Cancer communication: communication about cancer and cancer risk in the family on separate 4-point scales, information-seeking about health and cancer questions (yes/no), endorsements of type of information sought (National Cancer Institute [NCI], 2007) and interest in genetic testing for SC on a 5-point scale.

Skin cancer beliefs: assessment of SC preventability, diagnosis, and treatment from HINTS (NCI, 2007).

\section{Analysis}

Our goal was to examine differences among Hispanics and NHW in our domains of interest (SC risk reduction behaviors, communication, and beliefs). We examined differences between Hispanics and NHW on all variables using ChiSquare tests. Prior to regression analysis of the association between ethnicity and our dependent variables, we created low-high summary scores for each of the outcome variables (mean score for SC risk reduction behaviors, dichotomized then combined scores for cancer communication, and tally responses to nine SC belief statements for skin cancer beliefs). We included predictor variables (age, educational attainment, family SC history, and personal SC history) in the regression analysis that were related to ethnicity at the $\mathrm{p}<.10$ level in the original Chi-Square tests. These models were all performed using SPSS version 21 (Chicago, IL).

\section{Results}

Of the 104 participants recruited into the study, 48 that identified as Hispanic, and 36 as NHW were included in the analyses. On average Hispanics were less educated, less likely to report a family history and personal history of SC than NHW (data available from the authors).

Significant differences in SC risk reduction and screening behaviors were observed between Hispanics and NHW; Hispanics were significantly less likely to wear a long sleeve shirt while in the sun $(\mathrm{p}=0.01)$, and less likely 
to have received physician SC screening than NHW (p < 0.01; data available from the authors). There were no significant differences between Hispanics and NHW on SC communication with family as well as general cancer communication variables; in fact, endorsement of these items was quite high in both groups.

Both Hispanic and NHW participants frequently endorsed belief in the importance of early detection for SC (Figure 1). Yet, information overload ("It seems like everything causes skin cancer;" "It's hard to know which skin cancer recommendations to follow"), and other misconceptions about SC ("People with skin cancer would have pain prior to diagnosis") were more common among Hispanics. Hispanics were also significantly more likely than NHW to report that they would be reluctant to get checked for SC due to fears that they may have it. Conversely they were less likely to endorse the idea that the progression of $\mathrm{SC}$ can be slowed.

\section{Figure 1}

\section{Skin Cancer Beliefs in Hispanics and Non-Hispanic Whites}

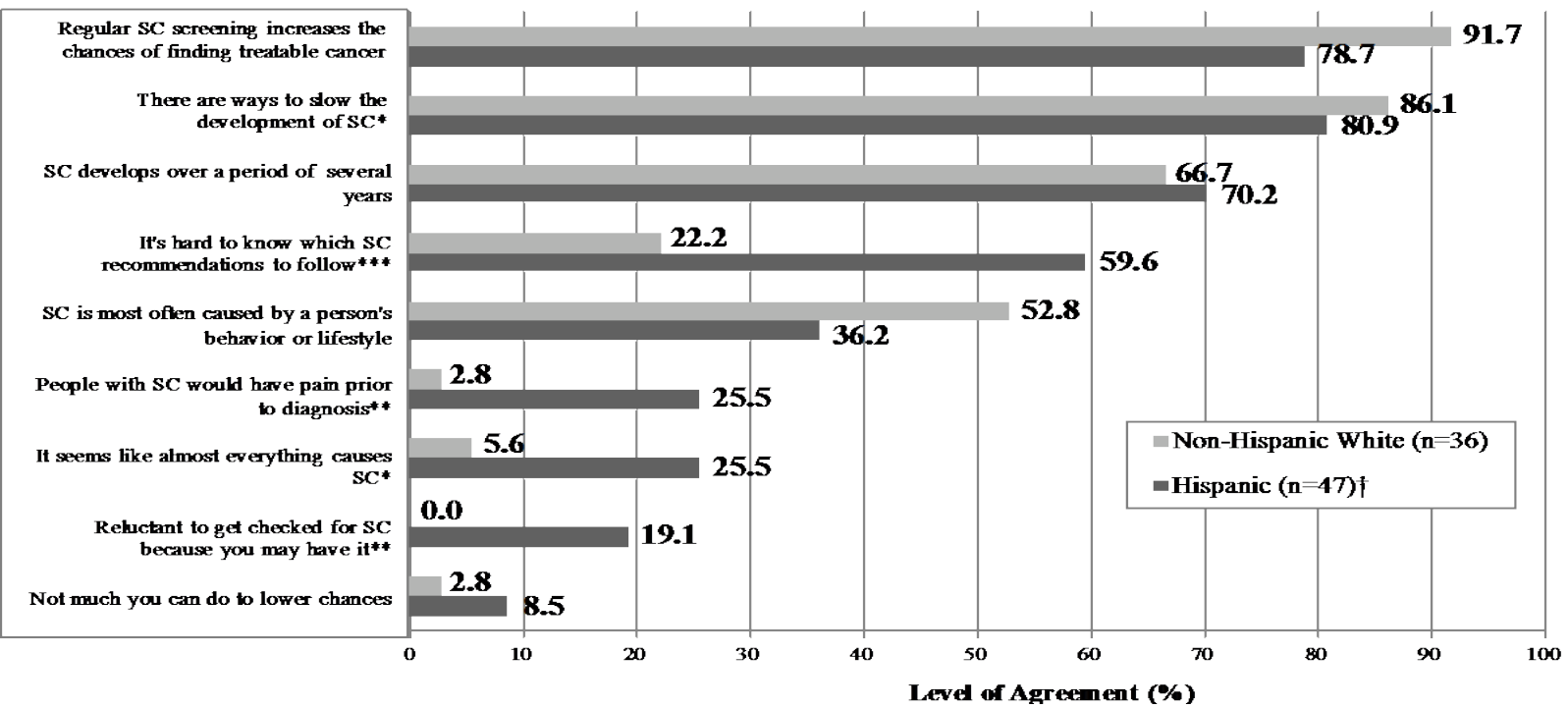

Level of percent agreement to several statements on skin cancer among Hispanics ( $\mathrm{n}=47)$ and Non-Hispanic Whites $(\mathrm{n}=36)$. Dark shaded square $=$ Hispanics, Lightly shaded square $=$ Non-Hispanic Whites. SC $=$ Skin cancer. ${ }^{*} p<$ $0.05 ; * * p<0.01, * * * p<0.001$. †One Hispanic participant skipped this section in its entirety.

Finally, we examined three multiple regression analyses examining our domains of interest (Table 1). In these adjusted models, we found significant differences when comparing SC beliefs across ethnicity (NHW versus Hispanic) and education ( $\leq$ high school versus $\geq$ College) categories. NHW reported more accurate SC beliefs than Hispanics. Participants with a college level education or greater reported more accurate SC beliefs than those with a high school education or less. In contrast, family history of SC was associated with increased SC risk reduction behaviors and cancer communication. An adjusted logistic regression model showed that neither ethnicity, nor any of the other predictor variables were related to screening (Results not shown). 
Torres S.M., Ramos M., Leverence R., Bowen D., Berwick M., Hay J. / Californian Journal of Health Promotion 2014, Volume 12, Issue 1, 95-100.

\section{Table 1}

\section{Regression Analyses with Skin Cancer Risk Reduction Behaviors, Cancer Communication, and SC} Beliefs as Outcomes $(\mathbf{N}=\mathbf{8 4})$

\begin{tabular}{|c|c|c|c|c|c|c|c|c|c|}
\hline \multirow[b]{2}{*}{ Variables } & \multicolumn{3}{|c|}{$\begin{array}{c}\text { SC Risk Reduction } \\
\text { Behaviors }\end{array}$} & \multicolumn{3}{|c|}{ Cancer Communication } & \multicolumn{3}{|c|}{ Skin Cancer Beliefs } \\
\hline & B & $\boldsymbol{\beta}$ & $p$-value & B & $\boldsymbol{\beta}$ & $p$-value & B & $\boldsymbol{\beta}$ & $p$-value \\
\hline Ethnicity $(\mathrm{NHW} / \mathrm{H})^{\mathrm{a}}$ & -0.09 & -0.06 & 0.61 & 0.02 & 0.01 & 0.96 & -1.11 & -0.36 & $0.01 * *$ \\
\hline Age & 0.01 & 0.19 & 0.09 & 0.02 & 0.21 & 0.07 & 0.01 & 0.01 & 0.99 \\
\hline Education $(\leq \text { HS } / \geq \text { College })^{b}$ & 0.27 & 0.18 & 0.09 & 0.13 & 0.05 & 0.68 & 1.12 & 0.36 & $0.01 * *$ \\
\hline Family History of SC (No/Yes) ${ }^{c}$ & 0.44 & 0.29 & $0.01 *$ & 0.94 & 0.33 & $0.01 * *$ & 0.41 & 0.13 & 0.21 \\
\hline Personal History of SC $(\mathrm{No} / \mathrm{Yes})^{\mathrm{c}}$ & 0.01 & 0.01 & 0.97 & 0.44 & 0.08 & 0.46 & -0.24 & -0.04 & 0.69 \\
\hline $\mathrm{R}^{2}$ & & 0.20 & & & 0.18 & & & 0.354 & \\
\hline $\mathrm{F}$ & & 3.65 & $0.01 * *$ & & 3.12 & $0.01 *$ & & 7.656 & $0.01 * * *$ \\
\hline $\begin{array}{l}{ }^{*} p<.05, * * p<.01, * * * p<.001 \\
\text { Abbreviations: Skin Cancer (SC); }\end{array}$ & Hispa & Whit & NHW); & $\operatorname{anic}(\mathrm{H}$ & gh scl & ol (HS) & & & \\
\hline
\end{tabular}

\section{Discussion}

Given that melanoma incidence and late-stage diagnosis is on the rise among some Hispanic populations (Rouhani et al., 2010), our research goals were to identify knowledge and behavior gaps that will dictate the need and content of educational materials tailored to Southwestern Hispanics. In general, we found very low levels of screening, which supports highlighting the importance of enhancing any opportunity to detect melanoma (Jaimes et al., 2013).

We found relatively high levels of existing communication about cancer in both groups. In prior work, Hispanics reported less exposure to health and cancer information than NHW (Hay et al., 2009). Our findings indicate that populations in the Southwest may be highly receptive to SC educational materials and that they may be motivated to discuss these materials with their families. One of the most important highlights of our findings is that our participants do not feel invulnerable to developing SC, which suggests receptivity to educational materials to address their potential risk.

Despite high levels of communication and information-seeking, we did identify higher levels of information overload and some misconceptions about SC in our Hispanic versus NHW groups. Over half of Hispanics endorsed some confusion about which SC recommendations to follow, and significant proportions of Hispanics compared to NHW agreed with the statement "it seems likely almost everything causes skin cancer" (24\%); and "people with skin cancer would have pain or other symptoms prior to diagnosis" (24\%). Similarly, in prior work we found NHW reported higher levels of awareness of accurate SC causes, prevention strategies and symptom knowledge, whereas Hispanics reported higher levels of information overload and misconceptions (Hay et al., 2009). While our sample included native born Hispanics, levels of health and cancer information-seeking, as well as misconceptions about cancer may be different among foreign born Hispanics. Accordingly, clear and manageable guidelines to address these beliefs would be useful and may need to be targeted to those at different levels of acculturation, who have likely not heretofore been the focus of public health education around SC.

\section{Limitations}

Our study has a number of important limitations. First, our pilot study findings will need to be confirmed in a larger sample. Second, our 
Torres S.M., Ramos M., Leverence R., Bowen D., Berwick M., Hay J. / Californian Journal of Health Promotion 2014, Volume 12, Issue 1, 95-100.

participants were clinic waiting room volunteers, thus we do not have information on nonvolunteers who may have differed on demographic or other variables of interest. Further, we did not collect information related to the purpose for their clinic visit; this may have impacted responses to the questionnaire if the visit was for any SC-related reason. It is possible that those who volunteered were more interested in SC-related topics, and thus could have been more highly-adherent to sun protection and screening with higher levels of informationseeking, than the clinic population in general. Some participants who reported that they were native born may not have wanted to reveal that they were foreign born. Third, we did not offer our survey in Spanish. About 20\% of the patient population seen at UNM clinics report that they are Spanish-speaking only, so we cannot generalize our findings to these patients. We did not assess the level of acculturation in our Hispanic study population; however, greater acculturation may shift sun protection in Hispanics (Heckman \& Cohen-Filipic, 2012), yet SC prevention interventions are likely needed for Hispanics regardless of acculturation level (Coups et al., 2013). Skin sensitivity information was not addressed in this study, but would be important to consider in further work as it is an important marker of SC risk, and thus need for sun protection strategies. However, there have not been any reports suggesting differences in sun protection among Hispanics after controlling for skin color and/or skin sensitivity to ultraviolet radiation. Finally, this pilot study was conducted in a medical clinic setting rather than a community-based survey, so it is not generalizable to those without access to the health care system who may likely have had greater SC misconceptions.

\section{Conclusions}

The U.S. Census Bureau (2013) estimates that by 2050, the proportion of Hispanics in the total population will rise from $15 \%$ to $30 \%$; further increasing the public health significance of melanoma as a problem in this population. Our findings lay the groundwork for the development of educational materials geared for English-speaking Albuquerque Hispanics to raise risk awareness regarding their susceptibility, knowledge of SC risk reduction and screening methods. Finally, such materials will be a useful basis for continued research and broader dissemination to other Hispanics across the United States in the future.

\section{Acknowledgements}

We acknowledge the support of NIH CA137532 and NIH CA133376 to Jennifer Hay; Supplement to RO1CA112524 (Marianne Berwick, PI) and NIGMS K12GM088021 to Salina Torres. The content of this manuscript is solely the responsibility of the authors and does not necessarily represent the official views of the National Institute of General Medical Sciences or the National Institutes of Health. Dr. Jennifer Hay had full access to the data in the study and takes responsibility for the integrity of the data and the accuracy of the data analysis.

\section{References}

American Cancer Society [ACS]. (2013). Skin Cancer Prevention and Early Detection. Available from: http://www.cancer.org/cancer/cancercauses/sunanduvexposure/skincancerpreventionandearlydete ction/skin-cancer-prevention-and-early-detection-intro. Accessed September 16, 2013.

Coups, E. J., Stapleton, J. L., Hudson, S. V., Medina-Forrester, A., Rosenberg, S. A., Gordon, M., et al. (2013). Skin cancer surveillance behaviors among US Hispanic adults. Journal of the American Academy of Dermatology, 68(4), 576-84.

Glanz K., Yaroch A. L., Dancel M., Saraiya M., Crane L. A., Buller D. B., et al. (2008). Measures of sun exposure and sun protection practices for behavioral and epidemiologic research. Archives of Dermatology, 144 (2), 217-222. 
Hay, J., Harris, J. N., Waters, E. A., Clayton, M. F., Ellington, L., Abernethy, A. D., \& Prayor-Patterson, H. (2009). Personal communication in primary and secondary cancer prevention: evolving discussions, emerging challenges. Journal of Health Communication, 14(1), 18-29.

Heckman, C. J., \& Cohen-Filipic, J. (2012). Brief Report: Ultraviolet radiation exposure, considering acculturation among Hispanics (Project URECAH). Journal of Cancer Education, 27(2), 342346.

National Cancer Institute [NCI]. (2007). Health Information National Trends Survey. Available from: http://hints.cancer.gov. Accessed July 1, 2013, September 16, 2013.

New Mexico Tumor Registry [NMTR]. (2011). New Mexico Cancer Statistics. Available from: http://hsc.unm.edu/som/nmtr/docs/CancerStatistics/1.10.110/Top\%20Sites_11JAN2011.pdf. Accessed July 1, 2013, September 16, 2013.

Jaimes, N., Oliveria, S., \& Halpern, A. (2013). A cautionary note on melanoma screening in the Hispanic/Latino population. JAMA Dermatology, 149(4), 396-397.

US Census Bureau. (2008). Hispanics in the United States. Available from: http://www.census.gov/population/hispanic/publications/hispanics_2006.html. Accessed July 1, 2013, September 16, 2013.

Weinstein, N. D. (1980). Unrealistic Optimism About Future Life Events. Journal of Personality and Social

Psychology, 39(5),

806-820.

Author Information

Salina M. Torres PhD, MPH

Robert Leverence MD

Marianne Berwick

Division of Epidemiology and Biostatistics, University of

New Mexico

Marcel Ramos BA

Department of Psychiatry \& Behavioral Sciences, Memorial

Sloan Kettering Cancer Center

Deborah Bowen $\mathrm{PhD}$

Department of Community Health Sciences, Boston

University School of Public Health

*Jennifer Hay PhD

Memorial Sloan-Kettering Cancer Center

Department of Psychiatry and Behavioral Sciences

641 Lexington Ave, 7th Floor New York, NY 10022,

Phone: (646) 888-0039

Email: hayj@mskcc.org.

* corresponding author 